\title{
Extralymphatic virus sanctuaries as a consequence of potent T-cell activation
}

\author{
Mike Recher ${ }^{1,10}$, Karl S Lang ${ }^{1,10}$, Alexander Navarini ${ }^{1,10}$, Lukas Hunziker ${ }^{1,2,10}$, Philipp A Lang ${ }^{1}$,
} Katja Fink ${ }^{1}$, Stefan Freigang ${ }^{1}$, Panco Georgiev ${ }^{3}$, Lars Hangartner ${ }^{1}$, Raphael Zellweger ${ }^{1}$, Andreas Bergthaler ${ }^{1}$, Ahmed N Hegazy ${ }^{1,4}$, Bruno Eschli ${ }^{1}$, Alexandre Theocharides ${ }^{5}$, Lukas T Jeker ${ }^{6}$, Doron Merkler ${ }^{1,7}$, Bernhard Odermatt $^{8}$, Martin Hersberger ${ }^{9}$, Hans Hengartner ${ }^{1}$ \& Rolf M Zinkernagel ${ }^{1}$

T helper cells can support the functions of $\mathrm{CDB}^{+} \mathrm{T}$ cells against persistently infecting viruses such as murine lymphocytic choriomeningitis virus (LCMV), cytomegalovirus, hepatitis C virus and HIV. These viruses often resist complete elimination and remain detectable at sanctuary sites, such as the kidneys and other extralymphatic organs. The mechanisms underlying this persistence are not well understood. Here we show that mice with potent virus-specific T-cell responses have reduced levels and delayed formation of neutralizing antibodies, and these mice fail to clear LCMV from extralymphatic epithelia. Transfer of virusspecific B cells but not virus-specific T cells augmented virus clearance from persistent sites. Virus elimination from the kidneys was associated with the formation of IgG deposits in the interstitial space, presumably from kidney-infiltrating $B$ cells. $C D 8^{+}{ }^{T}$ cells in the kidneys of mice that did not clear virus from this site were activated but showed evidence of exhaustion. Thus, we conclude that in this model of infection, site-specific virus persistence develops as a consequence of potent immune activation coupled with reductions in virus-specific neutralizing antibodies. Our results suggest that sanctuary-site formation depends both on organ anatomy and on the induction of different adaptive immune effector mechanisms. Boosting T-cell responses alone may not reduce virus persistence.

Some viruses are prone to developing persistent infections despite the presence of virus-specific immune responses ${ }^{1}$. Identified mechanisms of persistence include mutational escape from T-cell responses, resistance to interferons, expression of cytokine decoy receptors, inhibition of the cellular antigen-presentation machinery, glycan shielding of surface glycoproteins and integration into the host's genome $\mathrm{e}^{2-5}$. Virus sanctuaries-persistence of virus in extralymphatic tissues-not only complicate virus elimination, they also enable reactivation during immune suppression (for example, after organ transplantation) ${ }^{6}$. Virus persistence in certain tissues is partly restricted by the expression of specific receptors on tissue target cells; however, classical lymphotropic and/or hepatotropic viruses tend to persist in extralymphatic organs such as the kidney, brain, lungs, testes and salivary glands $s^{7-12}$. Persistence in these organs cannot always be explained by selective receptor expression; it also cannot be due simply to continuous virus spillover from the blood, as peripheral virus isolates differ phylogenetically from blood virus isolates and extralymphatic virus has been detected in individuals without viremia ${ }^{13}$.
Virus persistence and distribution are also shaped by innate resistance mechanisms $\mathrm{s}^{14,15}$ and drug therapy ${ }^{16}$. T helper cells are known to support $\mathrm{CD} 8^{+} \mathrm{T}$-cell responses that destroy cells infected with persisting viruses such as HIV, hepatitis C virus (HCV), cytomegalovirus (CMV) and LCMV ${ }^{17-19}$. However, the outcome of chronic HIV, HCV or CMV infections cannot always be predicted from T-cell response $^{20-22}$. Paradoxically, potent immune activation is a risk factor for end-stage disease in $\mathrm{HIV}^{23}$ and simian immunodeficiency (SIV) infections ${ }^{24}$ and has been associated with disease severity in cases of human influenza virus and severe acute respiratory syndrome (SARS)coronavirus infection ${ }^{25,26}$. Experimental immunization with T helper epitopes has even been found to worsen the outcome of SIV infection in rhesus macaques ${ }^{27}$. We and others have recently described $\mathrm{T}$ helper cell-driven suppression of neutralizing antibody formation after LCMV infection ${ }^{28,29}$. These findings together indicate that potent immune activation, which is usually beneficial, can sometimes be disadvantageous. To help delineate the mechanisms that influence this equilibrium, we have investigated the roles of specific $\mathrm{T}$ cells and

\footnotetext{
${ }^{1}$ Institute for Experimental Immunology, University Hospital Zürich, Schmelzbergstrasse 12, CH-8091 Zürich, Switzerland. ${ }^{2}$ Department for Internal Medicine, University Hospital Basel, 4031 Basel, Switzerland. ${ }^{3}$ Department of Visceral and Transplantation Surgery, University Hospital Zürich, Rämistrasse 100, 8091 Zürich, Switzerland. ${ }^{4}$ German Rheumatology Research Center, Charité Platz 1, D-10117 Berlin, Germany. ${ }^{5}$ Experimental Hematology, Department of Research, Basel University Hospital, 4031 Basel, Switzerland. 'Pediatric Immunology, Center for Biomedicine, University of Basel and University Children's Hospital of Basel, Mattenstrasse 28, 4058 Basel, Switzerland and Transplantation Immunology and Nephrology, University Hospital Basel, 4031 Basel, Switzerland. 7 Department of Neuropathology, Georg August University, Goettingen, Germany. ${ }^{8}$ Department of Pathology, University Hospital, Schmelzbergstrasse 12, CH-8091 Zürich, Switzerland. ${ }^{9}$ Institute of Clinical Chemistry, University Hospital Zürich, Rämistrasse 100, CH-8091 Zürich, Switzerland. ${ }^{10}$ These authors contributed equally to this work. Correspondence should be addressed to M.R. (rechermike@bluewin.ch).
}

Received 13 August; accepted 25 September; published online 4 November 2007; doi:10.1038/nm1670 

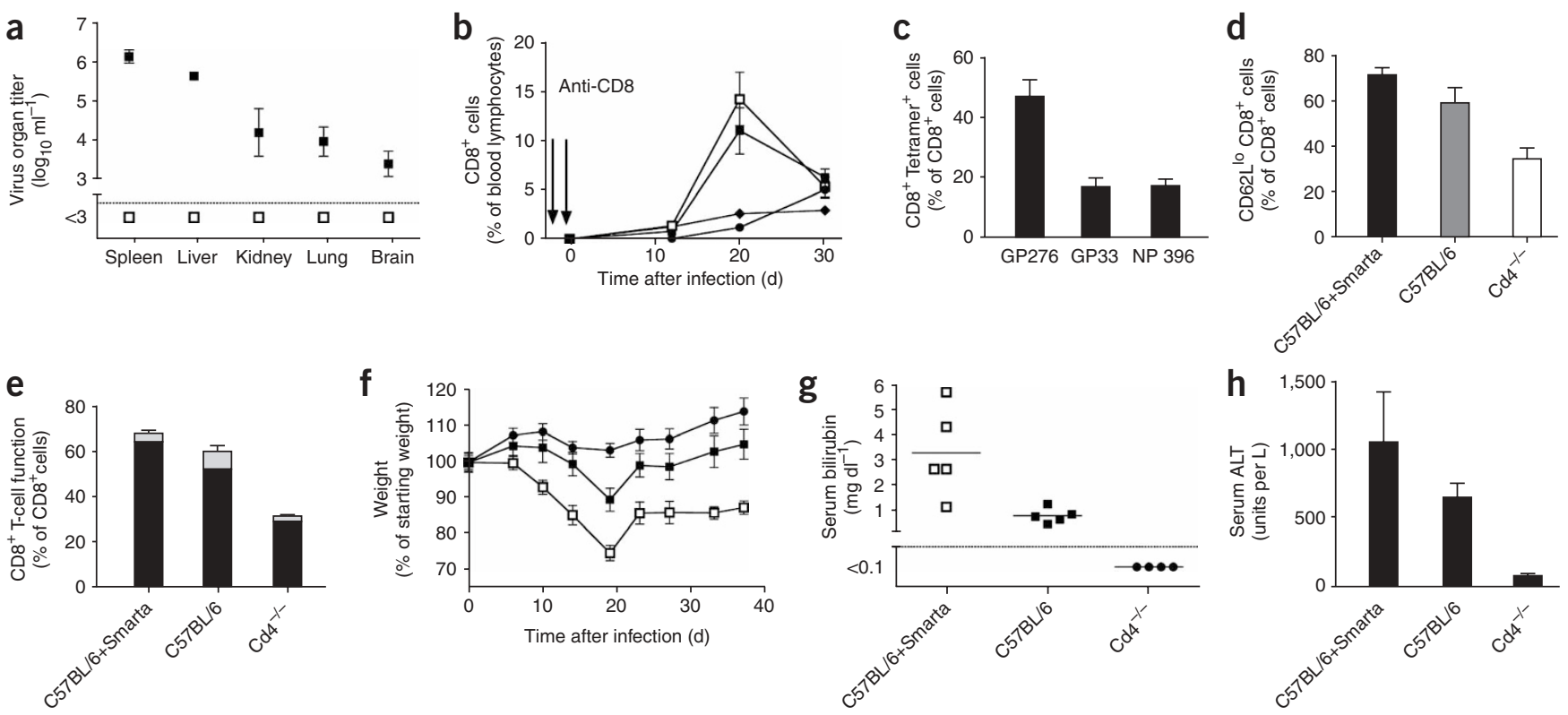

Figure 1 T helper cell-dependent CD8 ${ }^{+}$T-cell response against distributed virus. (a) C57BL/6 mice were depleted of CD8 $8^{+} \mathrm{T}$ cells at days -2 and -1 ( $\square$ ) or left untreated $(\square)$ before infection with 200 PFU LCMV-WE. Organ viral titers were measured $10 \mathrm{~d}$ later $(n=3)$. (b) C57BL/6 mice ( $\mathbf{\square})$, C57BL/6 mice supplemented with $10^{7}$ Smarta splenocytes on day -1 before infection (C57BL/6+Smarta, $\square$ ) or $\mathrm{Cd}^{-/}{ }^{-}$mice $(\bullet)$ were all transiently CD8 ${ }^{+} \mathrm{T}-\mathrm{cell}$ depleted with monoclonal antibodies administered on days -2 and -1 before infection, then infected with 200 PFU LCMV-WE on day 0 or left uninfected ( $)$ ). $\mathrm{CD}^{+} \mathrm{T}$ cells in peripheral blood were monitored at the indicated time points by FACS analysis $(n=5-10)$. (c) Twenty days after infection of C57BL/6 mice as described above, reemerging $C D 8^{+} T$ cells were tested for LCMV specificity using tetramer staining for three immunodominant epitopes (GP33-41, GP276-284 and NP396-404) and FACS analysis $(n=4-9)$. (d,e) CD62L expression (d) or direct ex vivo granzyme B (black) and IFN- $\gamma$ (gray) expression (e) of CD8 ${ }^{+}$T cells were measured by FACS $15 \mathrm{~d}(\mathbf{d})$ or $20 \mathrm{~d}(\mathbf{e})$ after infection $(n=5)$. (f-h) C57BL/6 mice ( $\left.\mathbf{\square}\right)$, C57BL/6+Smarta mice ( $\square$ ) or $\mathrm{Cd}^{-/}$mice $(\bullet)$ were infected with LCMV according to the standard protocol $(n=4-5)$. Mouse body weight was measured at the indicated time points (f). (g,h) $20 \mathrm{~d}$ after LCMV infection, serum bilirubin concentrations and serum amino liver transaminase (ALT) activity were measured $(n=5)$.

protective antibodies in enhancing or preventing the formation of extralymphatic persisting virus sanctuaries.

\section{RESULTS}

Induction and function of $\mathrm{CD}^{+} \mathrm{T}$ cells depends on $\mathrm{T}$ helper cells C57BL/6 mice infected with low-dose (200 plaque-forming units (PFU)) LCMV (WE strain), rapidly cleared the virus from all organs tested within $10 \mathrm{~d}$ (Fig. 1a). Infection of extralymphatic organs was absent or low, even at the peak of virus replication. To allow the virus to spread widely in the mouse before the onset of the $\mathrm{CD}^{+} \mathrm{T}$ cellderived immune response, we transiently depleted $\mathrm{CD}^{+} \mathrm{T}$ cells by administering monoclonal antibodies 1 or $2 \mathrm{~d}$ before infection (that is, on day -2 or day -1 ) with LCMV-WE (standard protocol, used throughout this manuscript). Ten days after infection, mice that had been depleted of $\mathrm{CD}^{+} \mathrm{T}$ cells showed virus replication in all organs tested (Fig. 1a). After their initial depletion, $\mathrm{CD}^{+} \mathrm{T}$ cells reemerged in the blood around day 10 after infection and then expanded, peaking at day 20 after infection as assessed by fluorescence-activated cell sorting (FACS) analysis of peripheral blood (Fig. 1b). This expansion of $\mathrm{CD}^{+} \mathrm{T}$ cells was almost completely virus specific, as up to $80 \%$ of these $\mathrm{CD}^{+} \mathrm{T}$ cells were tetramer-specific for known immunodominant virus epitopes (GP33-41, GP276-284 and NP396-404) (Fig. 1c; for representative FACS plot, see Supplementary Fig. 1a online). The $\mathrm{CD}^{+} \mathrm{T}$ cells were mostly of a CD62 $\mathrm{L}^{\text {lo }}$ phenotype, indicating activation (Fig. 1d). Most CD8 ${ }^{+} \mathrm{T}$ cells expressed granzyme B, and a small fraction produced interferon- $\gamma$ (IFN- $\gamma$ ) directly ex vivo without further restimulation (Fig. 1e; for representative FACS plots see Supplementary Fig. 1b). To determine the importance of $\mathrm{T}$ helper cells in $\mathrm{CD}^{+}$T-cell priming and proliferation in our model, we infected $C d 4^{--}$mice with 200 PFU LCMV-WE using the standard protocol described above. Expansion of $\mathrm{CD}^{+} \mathrm{T}$ cells in peripheral blood, as detected by FACS, was suppressed in $C d 4^{-/}$mice (Fig. 1b). $\mathrm{CD}^{+} \mathrm{T}$ cells were less activated, as downregulation of the selectin CD62L was impaired (Fig. 1d). Granzyme B-expressing $\mathrm{CD}^{+} \mathrm{T}$ cells were also relatively reduced in the absence of $\mathrm{T}$ helper cells (Fig. 1e and Supplementary Fig. 1b). By contrast, adoptive transfer of naive, virus-specific $\mathrm{T}$-cell receptor transgenic $\mathrm{CD} 4^{+} \mathrm{T}$ helper cells (Smarta cell $^{30}$ ) before LCMV infection of C57BL/6 mice (C57BL/6+Smarta) enhanced $\mathrm{CD}^{+} \mathrm{T}$-cell activation. CD62L downregulation and direct ex vivo granzyme $\mathrm{B}$ expression were both greater than in control C57BL/6 mice (Fig. 1d,e). Transfer of Smarta T helper cells augmented formation of IFN- $\gamma$ by T helper cells (Supplementary Fig. 1c). At the same time that $\mathrm{CD}^{+} \mathrm{T}$ cells peaked in mouse blood samples, the mice lost weight (Fig. 1f) and developed hepatitis, as indicated by elevated serum bilirubin concentrations and augmented liver transaminase activity in serum (Fig. 1g,h). Both weight loss and hepatitis correlated markedly with the number of available $\mathrm{T}$ helper cells (Fig. 1f-h). Thus, $\mathrm{CD}^{+} \mathrm{T}$-cell expansion and expression of effector functions (granzyme B and IFN $\gamma$ ) correlated with available T-cell help.

\section{Delayed virus clearance in mice with potent T-cell responses}

As expected, $\mathrm{Cd}^{-/}$mice infected with LCMV showed high viral titers in blood at all time points measured (Fig. 2a). However, virus elimination from the blood was, paradoxically, delayed in mice with high T-helper cell responses (C57BL/6+Smarta) compared with control C57BL/6 mice (Fig. 2a). The impaired virus clearance in mice 
a b

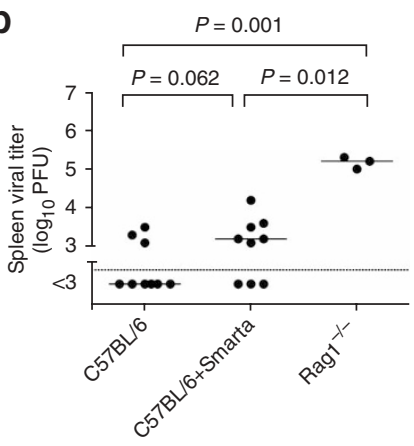

e

Figure 2 Organ-specific viral persistence enhanced by potent cellular immune responses. (a) Viral blood titers of C57BL/6 mice ( $\mathbf{\square})$, C57BL/6 mice supplemented with Smarta T helper cells (C57BL/6+Smarta, $\square$ ) or $\mathrm{Cd}^{-/}$mice infected with LCMV according to the standard protocol $(\bullet)$ were analyzed at the indicated time points $(n=3-5)$. (b-f) Twenty days after LCMV infection using the standard protocol, viral titers in the spleen (b), liver (c), brain (d), kidneys (e) and lungs (f) were measured in individual $\mathrm{Rag}^{-/}$mice, C57BL/6 mice and C57BL/6+Smarta mice as indicated (individual values are shown).

with potent T-cell responses was mostly due to a failure to clear the virus from the kidneys and lungs (Fig. 2b-f). Compared to Rag1 $1^{-/}$ mice, which are deficient in $\mathrm{T}$ cells and $\mathrm{B}$ cells, virus clearance was 1,000-10,000 times greater in the spleen and liver of C57BL/6+Smarta mice, although no significant difference was measured in the kidneys and lungs (Fig. 2b-f). The difficulty in eliminating the virus from the kidneys was still observed $50 \mathrm{~d}$ after infection of C57BL/6+Smarta mice, whereas virus control in the lungs and brain was comparable to that of control C57BL/6 mice at this time point (Supplementary Fig. 2a,b online). Notably, no virus could be detected in the blood at this time point (Fig. 2a). The observed site-specific virus elimination in C57BL/6+Smarta mice was not due to site-specific $\mathrm{T}$ helper cell responses, as $\mathrm{T}$ helper cell activation and function were comparable in the liver, kidneys and lungs (Supplementary Fig. 2c).
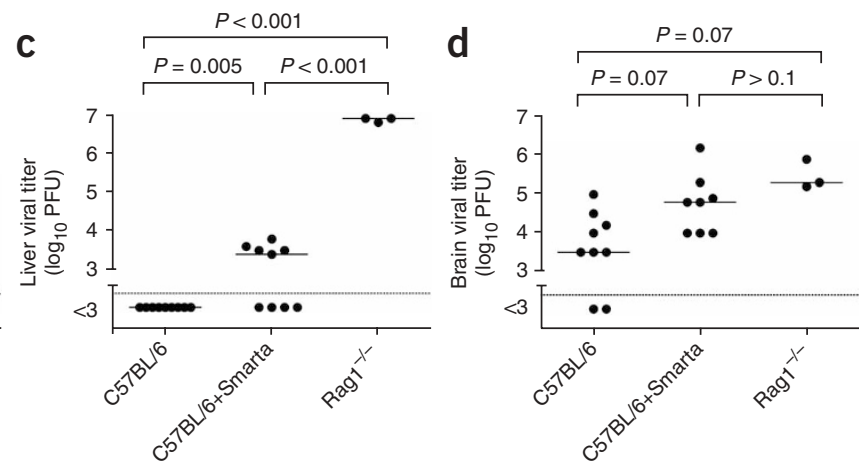

f

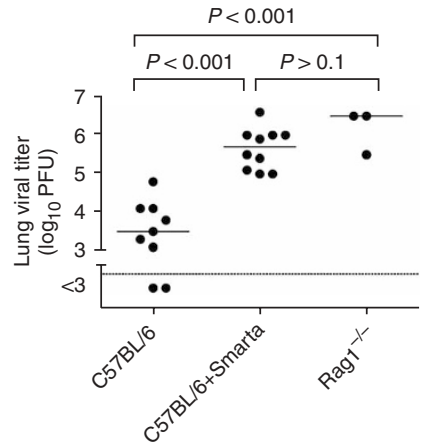

In summary, potent T-cell responses were apparently effective in lowering viral burden in some organs but did not clear virus from other tissues.

\section{Virus persistence correlates with delayed antibody formation}

LCMV-infected C57BL/6 mice developed measurable neutralizing antibodies by $30 \mathrm{~d}$ after infection (Fig. 3a). In keeping with our earlier results ${ }^{28}$, the formation of neutralizing antibodies was, paradoxically, delayed and reduced in mice supplemented with Smarta T helper cells, to a similar extent as in mice lacking $\mathrm{CD} 4^{+} \mathrm{T}$ cells (Fig. 3a). To test whether the delayed antibody formation was responsible for the observed site-specific virus persistence, we analyzed virus elimination in mice deficient in antibody formation. $\mathrm{Aic}_{\mathrm{C} \mathrm{a}^{-/}}$mice lack activationinduced cytidine deaminase (AID) and therefore do not undergo a

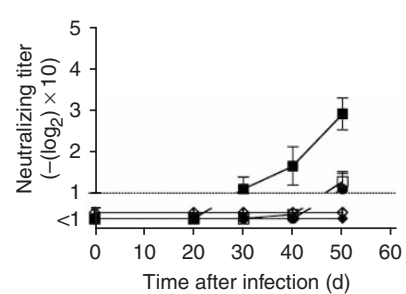

b

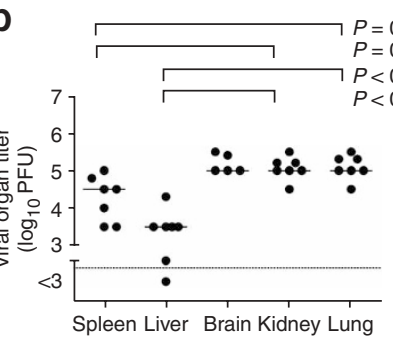

C

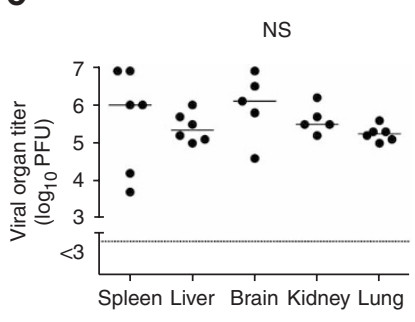

d

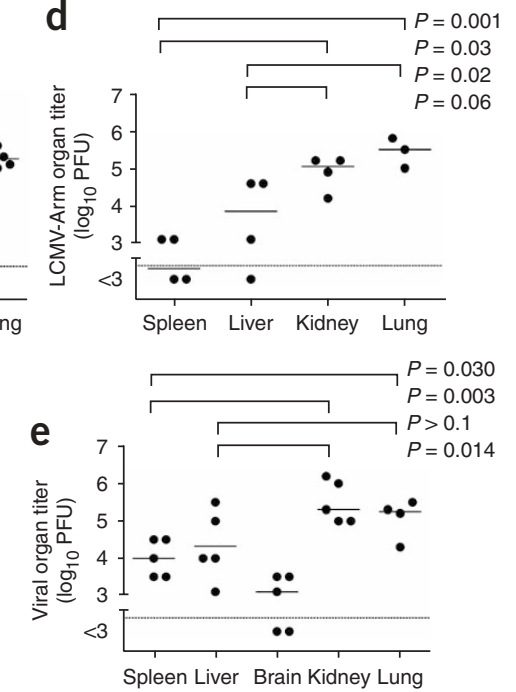

Figure 3 Site-specific viral persistence as a consequence of delayed antibody responses. (a) C57BL/6 mice ( $)$ ), C57BL/6 mice supplemented with transgenic virus-specific CD4 ${ }^{+}$T cells (C57BL/6+Smarta, $\square$ ), Cd4 ${ }^{-/}$ mice $(\bullet)$, Aicda ${ }^{-/}$mice $(\diamond)$ and VI1OYen mice $(\diamond)$ were depleted of CD8 $8^{+}$T cells on days -2 and -1 , then infected with 200 PFU LCMV-WE on day 0. (a) At the indicated time points, neutralizing antibody formation was assessed by neutralization assay $(n=4-9)$. (b-e) Aicda $^{-/}$mice were transiently depleted of $\mathrm{CD} 8^{+} \mathrm{T}$ cells on days -2 and -1 , then infected with 200 PFU LCMV-WE (b) or LCMV-Armstrong (LCMV-Arm) (d) on

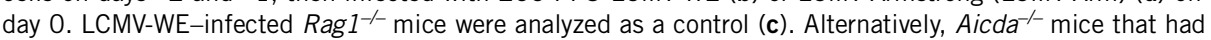
not been transiently depleted of CD8 $8^{+}$T-cells were infected with $2 \times 10^{6}$ PFU LCMV-WE (e). Viral organ titers were measured by plaque assay $30 \mathrm{~d}(\mathbf{b})$ or $50 \mathrm{~d}(\mathbf{c}, \mathbf{d}, \mathbf{e})$ after infection (individual values are shown). 
a

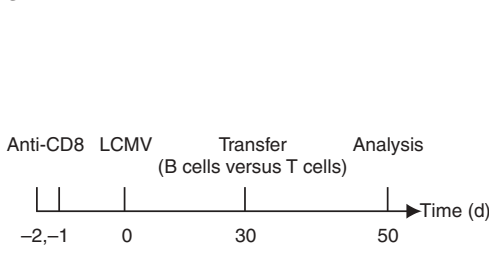

Figure 4 Reduction of extralymphatic virus sanctuaries associated with LCMV glycoprotein-specific B-cell responses. Aicda ${ }^{-/}$mice were transiently depleted of CD8 ${ }^{+} T$ cells on days -2 and -1 , then infected with 200 PFU LCMV-WE on day 0. Thirty days after infection, $1 \times 10^{7}$ LCMV-specific neutralizing B cells (+B cells) or LCMV-specific CD8 ${ }^{+} \mathrm{T}$ cells (+T cells) were adoptively transferred as described in the Methods. As controls, Aicda ${ }^{-/}$mice (without), VI10Yen mice (VI1OYen) or C57BL/6 mice (C57BL/6) were infected with LCMV according to the standard protocol but did not receive further adoptive cell transfer $30 \mathrm{~d}$ after infection. Vira organ titers were measured $50 \mathrm{~d}$ after LCMV infection. (a) Experimental setup. (b-f) Viral organ titers measured $50 \mathrm{~d}$ after LCMV infection in spleen (b), liver (c), brain (d), kidney (e) and lung (f). Individual values are shown.

immunoglobulin class switch recombination and affinity maturation, whereas other B-cell functions, including IgM responses, remain effective $^{31}$. LCMV-infected Aicda ${ }^{-/-}$mice did not produce neutralizing antibodies, as shown by a neutralization assay (Fig. 3a), and also did not produce LCMV glycoprotein-specific IgG responses, as tested by ELISAs (Supplementary Fig. 4b,c online). Notably, there were only slight differences in numbers of virus-specific $\mathrm{CD}^{+} \mathrm{T}$ cells, distribution of epitopes, production of IFN- $\gamma$, and $\mathrm{CD}^{+}$T-cell cytotoxicity between $\mathrm{Aicda}^{-/}$mice and C57BL/6 mice (Supplementary Fig. 3a-e online). Like $\mathrm{C} 57 \mathrm{Bl} / 6$ mice supplemented with Smarta cells, the LCMV-infected Aicda ${ }^{-/-}$mice showed site-specific virus persistence, as indicated by significantly lower viral titers in the spleen and liver $30 \mathrm{~d}$ after infection when compared to the kidneys, lungs and brain (Fig. 3b). In contrast, RAG-deficient mice had evenly distributed high viral titers in all organs tested (Fig. 3c). Site-specific persistence was also found in $\mathrm{Aicda^{-/- }}$ mice that were infected with another LCMV strain, LCMV-Armstrong (Fig. 3d), and in $\mathrm{Aicda}^{-/-}$ mice infected with high doses of LCMV-WE without initial transient $\mathrm{CD}^{+} \quad \mathrm{T}$-cell depletion (Fig. 3e).

\section{Reduction of virus sanctuaries by virus-specific B cells}

To analyze whether supplemented B cells were more effective than $\mathrm{T}$ cells at reducing viral titers, we adoptively transferred either LCMV-specific CD8 ${ }^{+} \mathrm{T}$ cells or neutralizing LCMV-specific B cells (see Methods) $30 \mathrm{~d}$ after LCMV infection of $\mathrm{Aicda}^{-/}$mice (for blood virus kinetics, see Supplementary Fig. 4a). Viral organ titers were measured 20 d later (see experimental schedule,
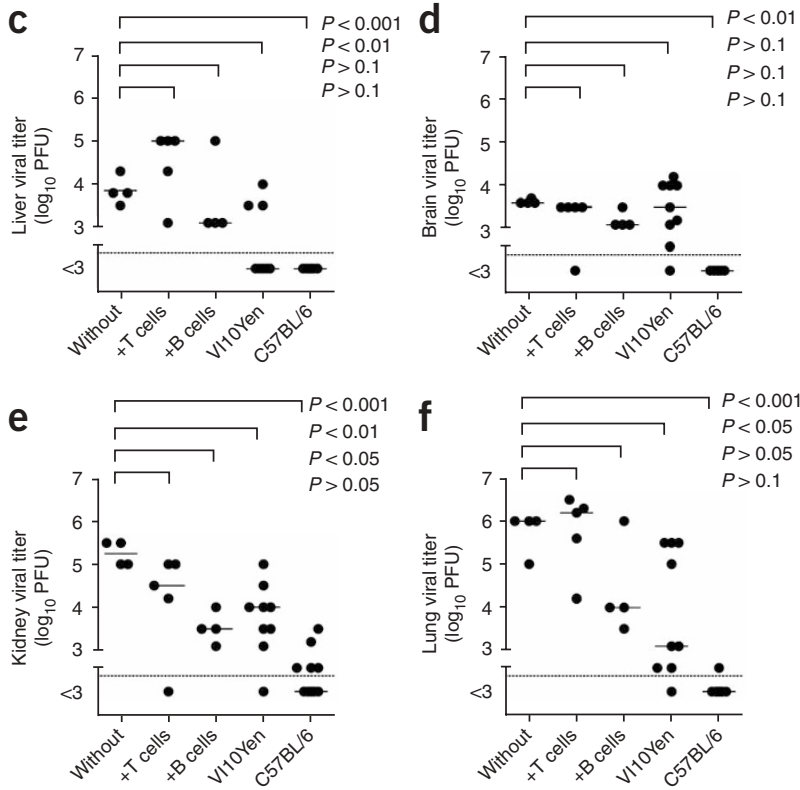

Fig. 4a). Fifty days after LCMV infection, $\mathrm{Aicda}^{-/}$mice without cell transfer showed the lowest viral titers in the liver and brain but had high titers in the spleen, lungs and kidneys (Fig. $\mathbf{4 b}-\mathbf{f}$ ). The increase in spleen viral titers at day 50 compared to day 30 is probably due to $\mathrm{CD}^{+}$T-cell exhaustion, which has been described to occur more rapidly in lymphoid tissue than in other organs ${ }^{32}$. Control C57BL/6 a

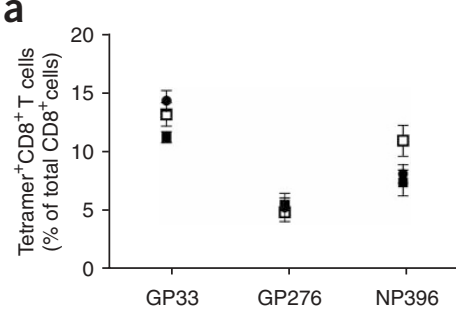

b
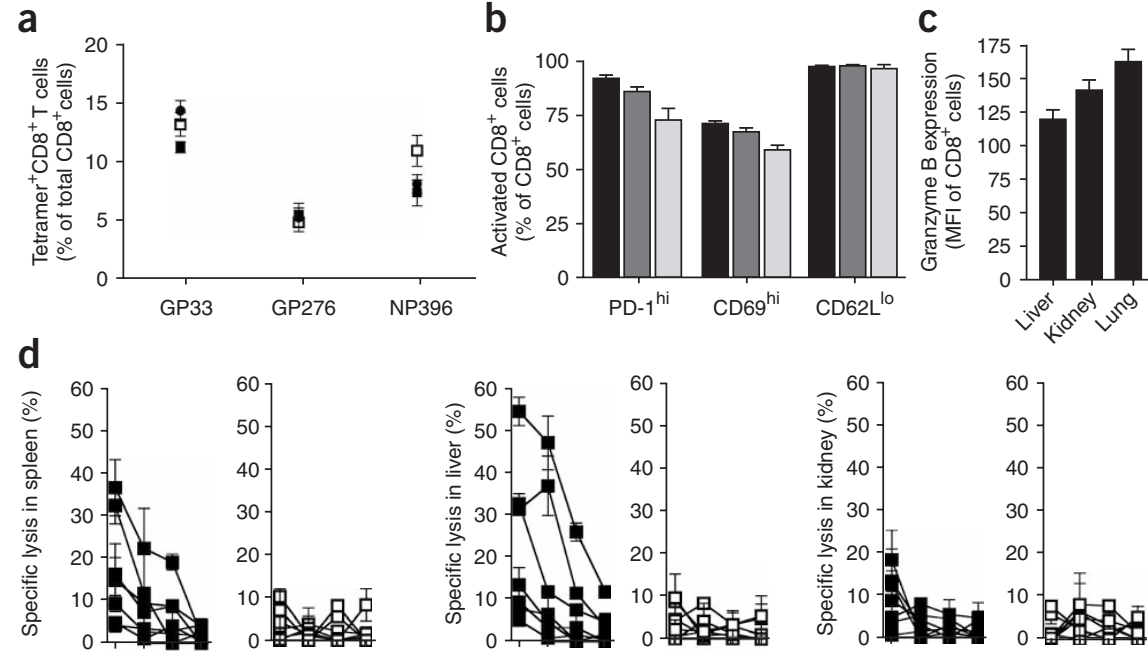

Threefold dilutions of effector cells (starting at effector/target ratio of 100:1)

Figure $5 \mathrm{~T}$ cells at sanctuary sites are activated but lack direct cytotoxicity. (a) Fifty days after infection using the standard protocol, virus was isolated from Aicda ${ }^{-1}$ mouse kidneys. We then injected 200 PFU of each of two isolates $(\square, \bullet)$ from two mice into separate naive C57BL/6 mice. As a control, we used 200 PFU of wild-type LCMV-WE ( $\mathbf{0}$ ). Ten days later, GP33-41, GP276-284 and NP396-404 tetramerpositive $\mathrm{CD} 8^{+}$T cells were measured by FACS analysis $(n=5)$. (b,c) Aicda ${ }^{-/}$mice were infected with LCMV according to the standard protocol. Thirty days after infection, CD8 ${ }^{+} \mathrm{T}$ cells isolated from the liver (black), kidneys (dark gray) or lungs (light gray) were assessed for expression of the indicated activation markers (b) and for granzyme B expression (c) by FACS analysis $(n=3-4)$. (d) Aicda $^{-/}$mice were infected with LCMV according to the standard protocol. Thirty days after infection, CD8 ${ }^{+} \mathrm{T}$ cells isolated from the indicated organs were analyzed for cytotoxicity in a chromium-release assay. Target cells were labeled with GP33-41 peptide $(\mathbf{\square})$ or unlabeled $(\square)$. Each line represents the mean and s.e.m. for one animal (chromium release measured in duplicates; $n=5-7$ experiments). 
Figure 6 Viral persistence at epithelial sites. (a) Fifty days after LCMV infection, $\operatorname{Rag} 1^{-/-}$mice, Aicda $^{-/}$mice with or without transient $\mathrm{CD} 8^{+}$ T-cell depletion, C57BL/6 mice, Smartasupplemented C57BL/6 mice (C57BL/6+Smarta) or VI1OYen mice were infected with LCMV using the standard protocol, immunohistology was used to detect LCMV nucleoprotein (VL4, i-vi), IgG (vii-xii) CD8 (xiii-xviii) and CD4 (xix-xxiv) in kidney sections $(n=2-5)$. (b) Aicda ${ }^{-/}$mice were LCMV infected with LCMV according to the

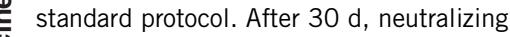
antibodies (monoclonal KL25 antibodies or polyclonal neutralizing serum) or LCMV-specific B cells were adoptively transferred. Twenty days later, expression of IgG, LCMV antigen (VL4) and plasma cells (CD138) was analyzed by immunohistology as indicated. (c, i) C57BL/ $6+$ Smarta mice were infected with LCMV according to the standard protocol. Fifty days later, immunohistological staining of LCMVnucleoprotein (VL4 antibody) was performed on sections of the distal urinary tract (one representative section is shown). (c, ii,iii) $\mathrm{Aicda}^{-1}$ mice were transiently depleted of $\mathrm{CD} 8^{+} \mathrm{T}$ cells on days -2 and -1 , then infected with 200 PFU LCMV-WE on day 0 . As a control, Rag $1^{-/}$mice

윽 were infected with LCMV on day 0 . Fifty days after infection, immunohistological staining of LCMV-nucleoprotein (VL4 antibody) was O. performed on liver sections (ii) or lung sections (iii). Scale bars, $200 \mu \mathrm{m}$.
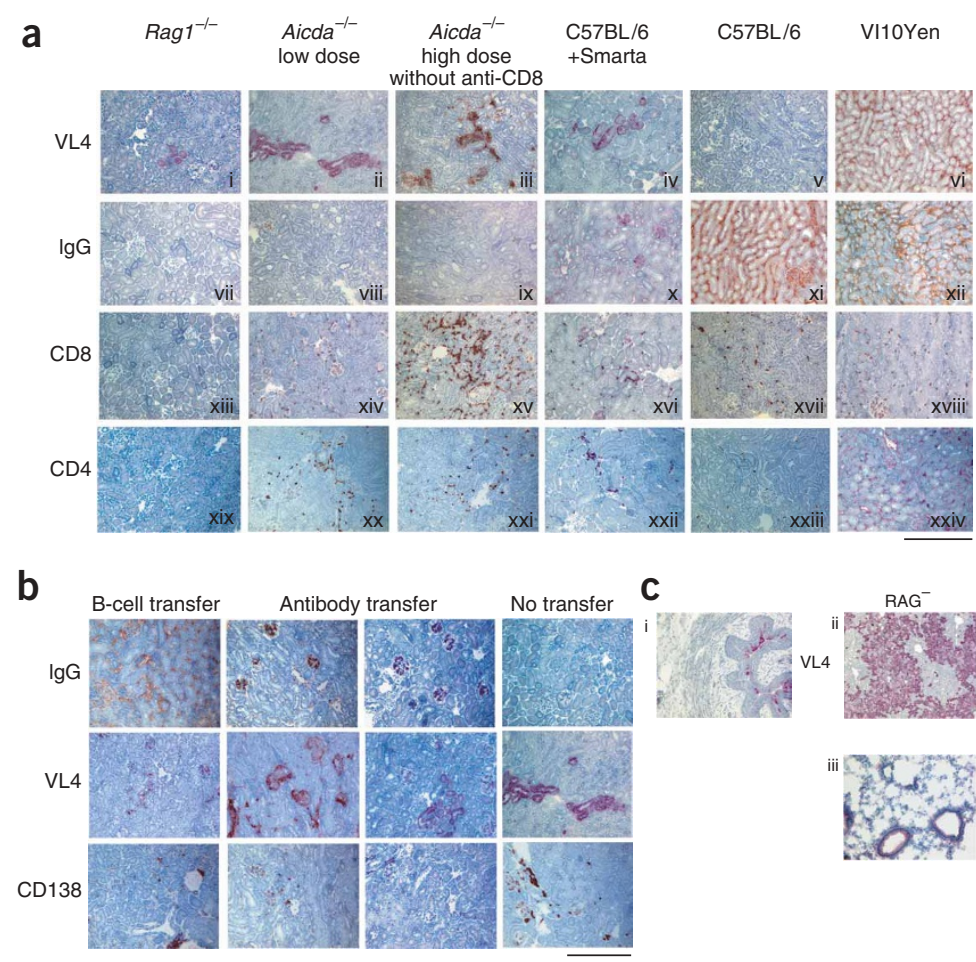

C
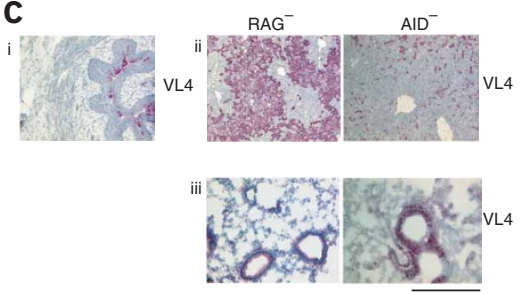

mice had almost completely cleared virus from all organs at this time point (Fig. 4b-f). LCMV-specific adoptive B-cell transfer into Aicda ${ }^{-/}$ mice was successful, as indicated by the rapid formation of LCMV glycoprotein-specific IgG (Supplementary Fig. $4 \mathbf{b}$ ), and was associated with significantly reduced viral titers in the kidneys and a trend toward lower titers in the lungs (Fig. 4e,f). By contrast, T-cell transfer did not significantly reduce viral titers in the kidneys or lungs (Fig. 4e,f). Transfer of LCMV glycoprotein-specific neutralizing antibodies into $\mathrm{Aicda}^{-/}$mice did not lower viral titers in the kidneys or lungs and did not induce measurable glycoprotein-specific IgG responses, implying that the antibodies were rapidly consumed by circulating virus particles (data not shown). We also analyzed LCMVinfected VI10Yen mice. In these mice, most naive B cells are specific for vesicular stomatitis virus (VSV) ${ }^{33}$. These mice did not produce LCMV-neutralizing antibodies for up to $50 \mathrm{~d}$ after infection (Fig. 3a); however, in contrast to AID-deficient mice, they eventually formed LCMV glycoprotein-specific IgG antibodies (Supplementary Fig. 4c). $\mathrm{CD}^{+}{ }^{+}$T-cell function in infected VI10Yen mice was comparable to that in C57BL/6 mice (data not shown). Compared to those in AID-deficient mice, viral titers in the kidneys were significantly lower in VI10Yen mice, mimicking the result of virus-specific B-cell transfer (Fig. $\mathbf{4 b - f )}$.

Together, these results show that the reduction of virus replication in some organs, such as the kidneys and lungs, correlates with the amount of LCMV glycoprotein-specific IgG antibodies but not with T-cell responses. Notably, B-cell transfer into $\mathrm{Aicda}^{-/-}$mice did not augment $\mathrm{CD} 8^{+} \mathrm{T}$-cell activation and function in extralymphatic organs (Supplementary Fig. 4d-f).

\section{Activated but exhausted $\mathrm{CD} 8^{+} \mathrm{T}$ cells in viral sanctuaries}

We next investigated the mechanisms that are involved in the formation of site-specific viral sanctuaries. First, we analyzed whether the persisting extralymphatic virus population consisted of $\mathrm{CD}^{+}{ }^{+} \mathrm{T}$-cell escape variants and thus resisted T-cell attack. Fifty days after infection using the standard protocol, we isolated the virus from the kidneys of two $A i c d a^{-/}$mice. We then injected 200 PFU of each kidney isolate or 200 PFU of wild-type LCMV-WE separately into naive C57BL/6 mice. Ten days later, we measured the expansion of LCMV GP33-41-specific, GP276-284-specific and NP396-404-specific CD8 ${ }^{+} \mathrm{T}$ cells by tetramer staining, finding only minimal differences (Fig. 5a). Lack of mutation was further confirmed by sequencing of one representative kidney isolate, which revealed LCMV-WE wild-type codon usage within and near the immunodominant GP276-284 epitope (data not shown).

Next, we compared the livers, kidneys and lungs of AID-deficient mice and found that $\mathrm{CD} 8^{+} \mathrm{T}$-cell activation and granzyme $\mathrm{B}$ expression were similar in these organs (Fig. $\mathbf{5 b}, \mathbf{c})$. Numbers of virus-specific $\mathrm{CD}^{+} \mathrm{T}$ cells and levels of IFN- $\gamma$ were also similar in the kidneys, lungs and liver (Supplementary Fig. $\mathbf{3 b}$,d), suggesting that differences in $\mathrm{CD}^{+}{ }^{+} \mathrm{T}$-cell activation and function could not explain the site-specific virus persistence observed in $\mathrm{Aicda}^{-/-}$mice.

In contrast, $\mathrm{CD}^{+}{ }^{+} \mathrm{T}$-cell cytotoxicity was lower in the kidneys than in the liver and spleen (Fig. 5d). Direct cytotoxicity correlated well with the measured viral titers in kidney versus liver and spleen at the same time point (Fig. 3). Thus, although we could not demonstrate differences in the intrinsic activation and function of $\mathrm{CD} 8^{+}$ $\mathrm{T}$ cells, the kidney $\mathrm{CD}^{+} \mathrm{T}$-cell population as a whole showed low cytotoxicity, which is indicative of exhaustion and associated with high viral kidney titers.

\section{Virus persistence at epithelial sites}

We performed immunohistological analysis of kidneys $50 \mathrm{~d}$ after infection of mice. In $\mathrm{Rag1}^{-/}$mice, which lack B and $\mathrm{T}$ cells, the virus was mainly located in the kidney tubule epithelium (Fig. 6a, i). 
Similar patterns of staining around the kidney tubules were observed in AID-deficient mice, either with or without transient $\mathrm{CD}^{+} \mathrm{T}$-cell depletion, and in mice transfused with Smarta T cells (Fig. 6a, ii,iii). Hence, despite a T-cell response, viral distribution in the kidneys remained the same as in Rag1 $1^{-/}$mice. By contrast, C57BL/6 mice had already efficiently eliminated the virus from their kidneys $50 \mathrm{~d}$ after infection (Fig. 6a, v). Extensive infiltrations of $\mathrm{CD}^{+} \mathrm{T}$ cells (Fig. 6a, xiii-xviii) and $\mathrm{CD}^{+} \mathrm{T}$ cells (Fig. 6a, xix-xxiv) were observed in the kidneys of all mice tested (except $\mathrm{T}$ cell-deficient $\mathrm{Rag}^{-/}$mice) independently of virus distribution, confirming that insufficient T-cell recruitment was not the reason for the epithelial persistence of virus antigen in $\mathrm{Aicda}^{-/}$mice and mice supplemented with Smarta $\mathrm{T}$ helper cells.

To further analyze virus elimination, we carried out immunohistological analysis of kidneys from VI10Yen mice, which had low but still detectable viral titers $50 \mathrm{~d}$ after infection (Fig. 4). In these mice, the virus was found mainly in the kidney interstitial space, rather than in epithelial cells. (Fig. 6a, vi). IgG staining in these mice localized to the same interstitial sites, supporting the idea that virus-antibody immune complexes form there (Fig. 6a, xii). Interstitial IgG deposition was also found in C57BL/6 mice, implying that interstitial IgG deposition remained after virus elimination (Fig. 6a, xi). As expected, IgG staining was not detectable in infected $\mathrm{Ragl}^{-/-}$mice and Aicda ${ }^{-/-}$ mice (Fig. 6a, vii-ix). When LCMV-neutralizing IgG was injected into infected $\mathrm{Aicda}^{-/}$mice IgG deposits were found mostly in kidney glomeruli, but usually not in the interstitial space surrounding the LCMV-infected epithelia (Fig. 6b), and LCMV kidney titers remained unchanged (data not shown). Thus, virus elimination from kidneys correlated with the formation of interstitial IgG deposits, presumably produced by kidney-infiltrating B cells. Plasma cells could easily be found within kidneys in immunohistological analyses (Fig. 6b). Kidney plasma cells were also observed in $\mathrm{Aicda}^{-/}$mice and C57BL/6 mice, whereas IgG deposition between kidney tubules was absent in $\mathrm{Aicda}^{-/-}$mice (Fig. 6b).

Virus was also detected in the lower urinary tract, where the epithelium is built of multiple cell layers. There, the virus was detected mainly in the upper cell layer (Fig. 6c), consistent with virus propagation in the urinary tract tubular system. Histological analysis of lung sections revealed similar virus sanctuaries in the bronchiolar epithelium in $\mathrm{Ragl}^{-/-}$mice and $\mathrm{Aicda}^{-/}$mice, indicating that lung epithelia might show resistance to T-cell attack similar to that seen in kidney epithelia (Fig. 6c). By contrast, in $\mathrm{Aicda}^{-/}$mice, which had low liver viral titers, the virus was found mainly in the Kupffer cell compartment of the liver, whereas virus was detected in liver hepatocytes in $\mathrm{Ragl}^{-/-}$mice (Fig. 3b and Fig. 6c).

\section{DISCUSSION}

Our experimental data mimic the paradoxical clinical finding that potent immune activation is sometimes a risk factor for viral persistence and severe disease. $\mathrm{CD}^{+} \mathrm{T}$ cells, supported by helper $\mathrm{T}$ cells, efficiently cleared the virus from some organs but not other organs. Virus was particularly persistent in the kidneys and lungs, but more efficiently cleared from the liver and spleen. We speculate that sitespecific viral persistence develops as a consequence of overactivated and exhausted T-cell responses combined with slow induction of virusspecific neutralizing antibodies. Such a combination is typical for several chronic human virus infections ${ }^{34}$. B cell-deficient mice with primed $\mathrm{T}$ cells specific for $\gamma$-herpesvirus do not eliminate the virus from the lungs, but do eliminate it from all other organs, upon reinfection $^{35}$. In addition, X-linked lymphoproliferative disease is similarly characterized by an overreactive cellular immune response combined with slow induction and low levels of virus-specific antibodies; this combination might lead to fatal outcomes of Epstein-Barr virus (EBV) infection in humans and of LCMV infection in mice $^{36}$. In our model of infection by LCMV, the $\mathrm{CD}^{+}{ }^{+}$T-cell response against distributed viral antigen was strengthened by the help of $\mathrm{CD}^{+} \mathrm{T}$ cells, as shown previously ${ }^{18}$. Similar findings exist for SIV and other persisting viruses ${ }^{17,19}$.

We found that the presence of virus-specific IgG responses was associated with virus clearance and with changes in virus distribution from epithelial cells to the interstitial space. Other models of persistent virus infection have similarly proposed that B-cell responses are associated with virus elimination ${ }^{37,38}$. Histological analysis showed that $\mathrm{T}$ cells efficiently infiltrated virus-infected epithelia, in a pattern reminiscent of 'autoreactivity' (for example, interstitial nephritis, bronchiolitis or Sjögren-like disease, which might convert into overt autoimmune disease). Although we did not detect CTL-escape variants at extralymphatic sites up to $50 \mathrm{~d}$ after infection, the observed colocalization of $\mathrm{CD}^{+} \mathrm{T}$ cells with the virus might eventually create sites conducive to the formation of CTL-escape mutants ${ }^{39}$. Lymphomas associated with chronic viral infection, which often arise in extralymphatic tissues, could be a consequence of ongoing extralymphatic immune responses. Together, our findings might explain why chronic virus infections, lymphomas and autoimmunity have been described as forming a triple association ${ }^{40}$.

In conclusion, the successful and complete elimination of a distributed persistence-prone viral infection requires efficient interplay between cellular and neutralizing humoral responses. Whereas T-cell responses can effectively clear the virus from both lymphatic and more 'solid' extralymphatic organs such as the liver, IgG responses are especially needed in organs with tubular epithelial microanatomy, such as the kidneys and lungs. The relative contributions of these two adaptive arms of the immune system also regulate immunopathological disease mediated by chronic inflammation. Thus, boosting of T-cell responses alone might not reduce virus persistence ${ }^{41}$ and might eventually induce immunopathology or autoimmunity ${ }^{36}$. Our results indicate that virus replication, tropism and sanctuary formation depend on the specific virus and on organ anatomy and physiology, but also on the induction, kinetics and efficacy of different adaptive immune effector mechanisms.

\section{METHODS}

Mice. All mice were bred and maintained under specific pathogen-free conditions, and experiments were performed in accordance with institutional and Swiss national guidelines. Animal care protocols were approved by the animal experiment committe of Zürich, Switzerland.

$C d 4^{-/-}$, VI10Yen-B-cell transgenic, $\operatorname{TgH}(\mathrm{KL} 25)-\mathrm{B}$-cell transgenic, 318-T-cell transgenic, Smarta1-T-cell transgenic, Ragl ${ }^{-/-}$and $\mathrm{Aicda}^{-/}$mice all have a C57BL/6 genetic background. All mice were purchased from the Institute for Labortierkunde, Faculty of Veterinary Medicine, University Zürich-Irchel.

Virus. LCMV strains WE and Armstrong (Arm) were originally gifts (see Acknowledgments) and were propagated on L929 or BHK21 cells. Mice were infected with $2 \times 10^{2}$ or $2 \times 10^{6} \mathrm{PFU}$ of LCMV injected intravenously as indicated.

Detection of viral and neutralizing antibody titers. LCMV viral titers were detected by a plaque-forming assay on MC57 fibroblasts as described ${ }^{42}$. Detection of neutralizing activity against LCMV in mouse sera has also been described $^{42}$. Briefly, neutralizing activity against LCMV-WE was measured in a plaque-reduction assay. The neutralizing titer was defined as the $\log _{2}$ dilution that caused a half-maximal reduction in plaques, with the reduction being measured in comparison to the number of plaques formed by similar amounts of the virus incubated with control sera from uninfected mice or medium alone. A titer of $<1$ indicates no detectable neutralization at an initial serum predilution of 1:10. 
In vivo depletion of $\mathrm{CD8}^{+} \mathrm{T}$ cells. Cell depletions were performed using rat monoclonal antibodies specific for CD8 (YTS 169.4) as described ${ }^{28}$. The hybridomas were initially obtained from H. Waldmann. We injected $200 \mu \mathrm{l}$ of purified $\mathrm{CD}^{+} \mathrm{T}$ cell-depleting antibodies intraperitoneally on days -2 and -1 before LCMV infection. The efficiency of depletion was always confirmed by FACS analysis of peripheral blood cells using fluorescein isothiocyanate (FITC)-, phycoerythrin (PE)-, or allophycocyanin (APC)labeled CD8-specific antibodies (Pharmingen).

Markers of liver injury. We measured serum activity of alanine aminotrans( ferase (ALT) as a marker of hepatocellular injury and total serum bilirubin concentrations using the serum multiple analyser (Ektachem DTSCII, Johnson and Johnson) or the Roche Modular Analytics Analyser with pyridoxal phosphate (Roche).

Adoptive cell transfer. $\mathrm{Aicda}^{-/-}$mice were transiently depleted of $\mathrm{CD} 8^{+} \mathrm{T}$ cells and infected with LCMV by the standard protocol, as described in Results. After $30 \mathrm{~d}$, either $1 \times 10^{7}$ MACS-sorted naive CD8 ${ }^{+} \mathrm{T}$ cells from LCMVspecific T-cell receptor-transgenic mice $\left(318\right.$ mice) or in vivo $\mathrm{CD}^{+} \mathrm{T}$ celldepleted naive TgHKL25 splenocytes (from LCMV-specific B cell-knock-in mice ${ }^{33}$ ) were adoptively transferred. Immunohistology and measurement of organ viral titers and $\mathrm{CD} 8^{+} \mathrm{T}$-cell function were performed $20 \mathrm{~d}$ later.

LCMV-neutralizing monoclonal antibodies (KL25, $1.5 \mathrm{mg}$ ) or polyclonal LCMV-neutralizing hyperimmune serum $(200 \mu \mathrm{l})$ were transfused intravenously as a control $30 \mathrm{~d}$ after LCMV infection of $\mathrm{Aicda}^{-/-}$mice.

윽 LCMV-glycoprotein GP1-specific IgG measurements. The detection of LCMV-glycoprotein GP-1-specific IgG by ELISA has been described ${ }^{28}$.

Fluorescence-activated cell sorting analysis of T-cell activation. Single-cell suspensions of the different organs were prepared. Cells were then incubated with fluorescent antibodies specific for PD-1 (PE-labeled, eBioscience), CD69 and CD62L (FITC-labeled, both BD Pharmingen), counterstained with fluorescent CD8-specific antibodies (APC-labeled, BD Pharmingen) and analyzed using a FACSCalibur system (BD Pharmingen). For $\mathrm{CD} 4^{+}$T-cell activation, antibodies to CD69, CD62L and CXCR3 (BD Pharmingen) were used.

For intracellular cytokine staining and T-cell restimulation, splenocytes were incubated for $5 \mathrm{~h}$ with or without LCMV peptide $(1 \mu \mathrm{M})$ or PMA $\left(50 \mathrm{ng} \mathrm{m}^{-1}\right)$ and ionomycin $\left(500 \mathrm{ng} \mathrm{m}^{-1}\right)$ for $6 \mathrm{~h}$ in RPMI medium supplemented with $10 \%$ FBS, $\beta$-mercaptoethanol and $5 \mu \mathrm{g} \mathrm{ml}^{-1}$ brefeldin A (Sigma-Aldrich). The cells were then harvested, washed once in PBS with $4 \%$ FBS and $12.5 \mathrm{mM}$ EDTA, and stained with PE- or APC-conjugated CD8-specific antibodies or PE-labeled antibodies specific for CD4 (BD Pharmingen). After washing, cells were fixed with $4 \%$ paraformaldehyde in PBS for $10 \mathrm{~min}$, then permeabilized using PBS with $4 \%$ FBS, $12.5 \mathrm{mM}$ EDTA and $0.1 \%$ saponin (Sigma-Aldrich). For intracellular cytokine staining, cells were incubated with PE-conjugated granzyme B-specific antibody (Caltag) or APC-labeled IFN- $\gamma$-specific antibody (BD Pharmingen) for $30 \mathrm{~min}$ at $4{ }^{\circ} \mathrm{C}$. After washing twice with permeabilization buffer, we resuspended the cells in PBS with 4\% FBS and 12.5 mM EDTA and analyzed them using a FACSCalibur system (BD Pharmingen).

In vivo cytotoxicity. In vivo cytotoxicity was assayed using C57BL/6 splenocytes incubated for $1 \mathrm{~h}$ with or without LCMV-derived MHC class I GP276-284 peptide and labeled for $10 \mathrm{~min}$ with $5 \mu \mathrm{g} \mathrm{ml}^{-1}$ carboxyfluorescein diacetate succinimidylester from Molecular Probes (CFSE ${ }^{\text {high }}$, peptide-labeled splenocytes) or $0.5 \mu \mathrm{g} \mathrm{m}^{-1}$ CFSE (CFSE ${ }^{\text {low, }}$, unlabeled splenocytes). We injected $10^{7}$ cells of each fraction intravenously into AID-deficient or control C57BL/6 mice $50 \mathrm{~d}$ after LCMV infection using the standard protocol described in Results. The number of CFSE-positive cells remaining in blood $12 \mathrm{~h}$ later was determined by FACS analysis. Specific cytotoxicity was calculated from the cell counts of LCMV-infected (memory) and noninfected (naive) mice under each treatment, as 1 - $\left(\mathrm{CFSE}^{\text {high }}{ }_{\text {memory }} / \mathrm{CFSE}^{\text {low }}{ }_{\text {memory }}\right) /\left(\mathrm{CFSE}^{\text {high }}{ }_{\text {naive }} / \mathrm{CFSE}^{\text {low }}{ }_{\text {naive }}\right)$, and expressed as a percentage.

${ }^{51} \mathrm{Cr}$-release cytotoxicity assay. We performed ${ }^{51} \mathrm{Cr}$ release assays as described $^{43}$. Single-cell preparations of organs were prepared and lymphocytes separated by gradient centrifugation (Lympholyte, Cedarlane). Threefold dilutions of effector cells with a starting effector/target ratio of 100:1 were analyzed.
Immunohistology. Histological analysis was performed on snap-frozen tissue sections stained with rat monoclonal antibodies against LCMV-nucleoprotein (VL4), CD8 (53-6.7), IgG (mixture of rat anti-mouse IgG1, IgG2a/b and IgG3) or CD138. Monoclonal antibodies were detected with goat anti-rat (Caltag) and alkaline phosphatase-labeled donkey anti-goat (Jackson ImmunoResearch) secondary antibodies, which we visualized by using naphthol 6-bromo-2hydroxy-3-naphtholic acid 2-methoxy anilide (AS-BI) phosphate and new fuchsin as a substrate. The enzyme reaction yielded a red reaction product. Kidney sections were stained in some experiments with goat anti-rat secondary antibody (Caltag), followed by donkey anti-goat labeled with horseradish peroxidase. Incubation with 3-amino-9-ethylcarbazole (AEC) in the presence of $\mathrm{H}_{2} \mathrm{O}_{2}$ yielded a brown reaction product. Sections were counterstained with hemalum.

Statistical analysis. Data are presented as mean \pm s.e.m. or as single values (median value visualized as a horizontal line). Values in treatment groups were analyzed for significant differences using a one-way analysis of variance followed by a Tukey least-significant difference test. For statistical analysis, organ viral titers below detection limits were set to $10^{1.5} \mathrm{PFU}$.

Note: Supplementary information is available on the Nature Medicine website.

\section{ACKNOWLEDGMENTS}

We thank A.J. MacPherson and D. Pinschewer for discussions and K. Tschannen for technical assistance. Aicda ${ }^{-/-}$mice were provided by T. Honjo (Kyoto University). LCMV strains WE and Armstrong (Arm) were originally obtained from F. Lehmann-Grube (Heinrich Pette Institute) and M. Buchmeier (Scripps Institute), respectively. This work was supported by Swiss National Foundation Grants to H.H. (3100A0-100779) and R.M.Z. (3100A0-100068). K.S.L. was partially supported by the Deutsche Forschungsgemeinschaft LA 1419/1-1. A.B. holds a PhD fellowship of the Boehringer Ingelheim Fonds. A.N.H. is a fellow of Graduate School 1121 of the German Science Foundation.

\section{AUTHOR CONTRIBUTIONS}

M.R., K.S.L., A.N. and L.H. planned and performed most experiments and wrote the manuscript; P.A.L. performed experiments during the revision process; S.F. performed some experiments with AID-deficient mice; A.B. sequenced persisting kidney virus; A.N.H. helped isolate lymphocytes for cytotoxicity experiments, K.F., L.H. and R.Z. helped perform experiments with B cell-transgenic mice and provided materials; B.E. helped perform LCMV glycoprotein-specific ELISAs and provided materials; D.M. did statistical testing; B.O. performed and supervised immunohistology; P.G. and M.H. performed and supervised measurement of serum hepatitis markers; A.T. and L.T.J. contributed to discussions and writing of the manuscript; and H.H. and R.M.Z. supervised and financed the project and helped write the manuscript.

Published online at http://www.nature.com/naturemedicine

Reprints and permissions information is available online at http://npg.nature.com/ reprintsandpermissions

1. Hilleman, M.R. Strategies and mechanisms for host and pathogen survival in acute and persistent viral infections. Proc. Natl. Acad. Sci. USA 101 Suppl. 2, 14560-14566 (2004).

2. Peterlin, B.M. \& Trono, D. Hide, shield and strike back: how HIV-infected cells avoid immune eradication. Nat. Rev. Immunol. 3, 97-107 (2003)

3. Alcami, A. Viral mimicry of cytokines, chemokines and their receptors. Nat. Rev. Immunol. 3, 36-50 (2003).

4. Wei, X. et al. Antibody neutralization and escape by HIV-1. Nature 422, 307-312 (2003).

5. Rehermann, B. \& Nascimbeni, M. Immunology of hepatitis B virus and hepatitis C virus infection. Nat. Rev. Immunol. 5, 215-229 (2005).

6. Fischer, S.A. et al. Transmission of lymphocytic choriomeningitis virus by organ transplantation. N. Engl. J. Med. 354, 2235-2249 (2006).

7. Bruggeman, L.A. et al. Renal epithelium is a previously unrecognized site of HIV-1 infection. J. Am. Soc. Nephrol. 11, 2079-2087 (2000).

8. Arrieta, J.J. et al. In situ detection of hepatitis $C$ virus RNA in salivary glands. Am. J. Pathol. 158, 259-264 (2001).

9. Becker, J.L. et al. Epstein-Barr virus infection of renal proximal tubule cells: possible role in chronic interstitial nephritis. J. Clin. Invest. 104, 1673-1681 (1999).

10. Stewart, J.P., Usherwood, E.J., Ross, A., Dyson, H. \& Nash, T. Lung epithelial cells are a major site of murine gammaherpesvirus persistence. J. Exp. Med. 187, 1941-1951 (1998).

11. Ahmed, R., Jamieson, B.D. \& Porter, D.D. Immune therapy of a persistent and disseminated viral infection. J. Virol. 61, 3920-3929 (1987). 
ARTICLES

12. Chang, H. et al. Human immunodeficiency virus type 1 in the semen of men receiving highly active antiretroviral therapy. N. Encl. J. Med. 339, 1803-1809 (1998).

13. Maras, D. et al. Replication and compartmentalization of HIV-1 in kidney epithelium of patients with HIV-associated nephropathy. Nat. Med. 8, 522-526 (2002).

14. Flodstrom, M. et al. Target cell defense prevents the development of diabetes after viral infection. Nat. Immunol. 3, 373-382 (2002).

15. Lang, K.S. et al. Toll-like receptor engagement converts T-cell autoreactivity into overt autoimmune disease. Nat. Med. 11, 138-145 (2005).

16. Wolf, D.G. et al. Emergence of late cytomegalovirus central nervous system disease in hematopoietic stem cell transplant recipients. Blood 101, 463-465 (2003).

17. Lichterfeld, M. et al. Loss of HIV-1-specific CD 8+ T cell proliferation after acute HIV-1 infection and restoration by vaccine-induced HIV-1-specific CD4+ T cells. J. Exp. Med. 200, 701-712 (2004).

18. Matloubian, M., Concepcion, R.J. \& Ahmed, R. CD4+ T cells are required to sustain CD8+ cytotoxic T-cell responses during chronic viral infection. J. Virol. 68, 80568063 (1994).

19. Grakoui, A. et al. HCV persistence and immune evasion in the absence of memory T cell help. Science 302, 659-662 (2003).

20. Kiepiela, P. et al. CD8(+) T-cell responses to different HIV proteins have discordant associations with viral load. Nat. Med. 13, 46-53 (2007).

21. Thomson, M. et al. The clearance of hepatitis $C$ virus infection in chimpanzees may not necessarily correlate with the appearance of acquired immunity. J. Virol. 77, 862-870 (2003).

22. Bronke, C., Westerlaken, G.H., Miedema, F., Tesselaar, K. \& van Baarle, D. Progression to CMV end-organ disease in HIV-1-infected individuals despite abundance of highly differentiated CMV-specific CD8+ T-cells. Immunol. Lett. 97, 215-224 (2005).

23. Sousa, A.E., Carneiro, J., Meier-Schellersheim, M., Grossman, Z. \& Victorino, R.M. CD4 T cell depletion is linked directly to immune activation in the pathogenesis of HIV -1 and HIV-2 but only indirectly to the viral load. J. Immunol. 169, 3400-3406 (2002).

24. Silvestri, G. et al. Nonpathogenic SIV infection of sooty mangabeys is characterized by limited bystander immunopathology despite chronic high-level viremia. Immunity 18, 441-452 (2003).

25. Kobasa, D. et al. Aberrant innate immune response in lethal infection of macaques with the 1918 influenza virus. Nature 445, 319-323 (2007).

26. Lo, A.W., Tang, N.L. \& To, K.F. How the SARS coronavirus causes disease: host or organism? J. Pathol. 208, 142-151 (2006).

27. Staprans, S.I. et al. Enhanced SIV replication and accelerated progression to AIDS in macaques primed to mount a CD4 T cell response to the SIV envelope protein. Proc. Natl. Acid. Sci. USA 101, 13026-13031 (2004).

28. Richer, M. et al. Deliberate removal of T cell help improves virus-neutralizing antibody production. Nat. Immunol. 5, 934-942 (2004).
29. Matter, M., Odermatt, B., Yagita, H., Nuoffer, J.M. \& Ochsenbein, A.F. Elimination of chronic viral infection by blocking CD27 signaling. J. Exp. Med. 203, 2145-2155 (2006).

30. Oxenius, A., Bachmann, M.F., Zinkernagel, R.M. \& Hengartner, H. Virus-specific MHCclass II-restricted TCR-transgenic mice: effects on humoral and cellular immune responses after viral infection. Eur. J. Immunol. 28, 390-400 (1998).

31. Harada, Y., Muramatsu, M., Shibata, T., Honjo, T. \& Kuroda, K. Unmutated immunoglobulin $\mathrm{M}$ can protect mice from death by influenza virus infection. J. Exp. Med. 197, 1779-1785 (2003).

32. Wang, X.Z. et al. Virus-specific CD 8 T cells in peripheral tissues are more resistant to apoptosis than those in lymphoid organs. Immunity 18, 631-642 (2003).

33. Hangartner, L. et al. Antiviral immune responses in gene-targeted mice expressing the immunoglobulin heavy chain of virus-neutralizing antibodies. Proc. Natl. Acad. Sci. USA 100, 12883-12888 (2003).

34. Legrand, E. et al. Course of specific T lymphocyte cytotoxicity, plasma and cellular viral loads, and neutralizing antibody titers in 17 recently seroconverted HIV type 1-infected patients. AIDS Res. Hum. Retroviruses 13, 1383-1394 (1997).

35. Andreansky, S. et al. The limits of protection by "memory" T cells in lg-/- mice persistently infected with a gamma-herpesvirus. Proc. Natl. Accad. Sci. USA 101, 2017-2022 (2004).

36. Grotty, S., McCausland, M.M., Aubert, R.D., Wherry, E.J. \& Ahmed, R. Hypogammaglobulinemia and exacerbated CD8 T cell mediated immunopathology in SAP-deficient mice with chronic LCMV infection mimics human XLP disease. Blood 108, 30853093 (2006).

37. Planz, O. et al. A critical role for neutralizing-antibody-producing B cells, CD4(+) T cells, and interferon in persistent and acute infections of mice with lymphocytic choriomeningitis virus: implications for adoptive immunotherapy of virus carriers. Proc. Natl. Accad. Sci. USA 94, 6874-6879 (1997).

38. Lauterbach, H., Truong, P. \& McGavern, D.B. Clearance of an immunosuppressive virus from the CNS coincides with immune reanimation and diversification. Virol. J. 4, 53 (2007).

39. Allen, T.M. et al. Tat-specific cytotoxic T lymphocytes select for SIV escape variants during resolution of primary viraemia. Nature 407, 386-390 (2000).

40. Ramos-Casals, M. et al. Triple association between hepatitis $C$ virus infection, systemic autoimmune diseases, and B cell lymphoma. J. Rheumatol. 31, 495-499 (2004).

41. Oxenius, A. et al. Stimulation of HIV-specific cellular immunity by structured treatment interruption fails to enhance viral control in chronic HIV infection. Proc. Natl. Acad. Sci. USA 99, 13747-13752 (2002).

42. Battegay, M. et al. Quantification of lymphocytic choriomeningitis virus with an immunological focus assay in 24- or 96-well plates. J. Tirol. Methods 33, 191-198 (1991).

43. Junt, T. et al. Antiviral immune responses in the absence of organized lymphoid T cell zones in plt/plt mice. J. Immunol. 168, 6032-6040 (2002).

일

NATURE MEDICINE VOLUME 13 | NUMBER 11 | NOVEMBER 2007

1323 